\title{
The Two Dimensional Nanoplate Dynamics Revealed by in situ Liquid Cell TEM
}

\author{
Xuezeng Tian ${ }^{1}$, Jingyu Lu², Zainul Aabdin ${ }^{2}$, Utkarsh Anand ${ }^{2}$, Utkur M. Mirsaidov and Haimei Zheng ${ }^{2}, 3$ \\ ${ }^{1 .}$ Berkeley Education Alliance for Research in Singapore, 138602, Singapore \\ 2. Center for BioImaging Sciences, Department of Biological Sciences, National University of Singapore, \\ Science Drive 4, Singapore 117543 \\ 3. Materials Sciences Division, Lawrence Berkeley National Laboratory, Berkeley, CA 94720
}

The interface between solid and liquid is an intriguing topic in many fields of studies. However, such a heterogeneous interface is not easy to probe using conventional methods, especially when the length at nanometre length scales. Recent progress in in situ TEM enables the study of physical and chemical processes at interfaces. A liquid cell usually consists of a thin liquid layer sandwiched between two electron transparent silicon nitride membranes. This allows the real time observation of interfacial phenomena with sub-nanometre resolutions. For example, the diffusivity of gold nanoparticles is damped due to interaction with the membrane ${ }^{[1][2]}$; the movement of a few nanometres water droplet is greatly influenced by the interfacial forces ${ }^{[3]}$. In this work, we use liquid cell in TEM to study conventional materials with unexpected new features. We'll show peculiar dynamics of $\mathrm{AgCl}$ nanoplates, which result from the interplay of surface tension and Coulomb repulsive force, whereas we use electron beam to control the balance between these two competing phenomenon.

The $\mathrm{AgCl}$ nanoplates were made by dissolving silver nanoparticles in $\mathrm{KCl}$ solution. Electron beam irradiation accelerates this dissolving process. All the $\mathrm{AgCl}$ in our experiments take a rock salt structure with lattice constant of $5.55 \AA$. We find that the $\mathrm{AgCl}$ particles prefer cubic shapes, with all the facets in $\{002\}$ planes. The liquid we use in the experiment is $25 \mathrm{mM} \mathrm{KCl}$ solution. To our best knowledge, when $\mathrm{AgCl}$ is immersed in $\mathrm{KCl}$ solution, the surface will preferentially be adsorbed by chloride ion due to the strong bonding of silver ions to chloride ions ${ }^{[4]}$. Thus the first adsorption layer of $\mathrm{AgCl}$ is dominated by negatively charged chloride ions whereas outer adsorption layers being balanced by positive potassium ions. Such an adsorption configuration results in both vertical and lateral potential differences. The lateral potential causes the Coulomb repulsive force which tends to expand the nanoparticles. However, the surface tension tends to contract the particles to lower its internal energy.

There is a balance between the surface tension and Coulomb repulsion. It's interesting to note that electron dose could mediate this balance and lead to completely different dynamics. Under low electron dose, the surface tension of $\mathrm{AgCl}$ nanocubes will be dominating. Thus the nanocubes are relaxed into spherical shape. Increasing the dose will change the adsorption equilibrium, which leads to larger Coulomb repulsion which leads to expansion of nanocubes into nanoplates. Further increasing the dose causes the balance between surface tension and Coulomb repulsion will be broken. Consecutively, $\mathrm{AgCl}$ nanoplates split up into smaller pieces. Fig. 1 shows a typical splitting event. During this splitting event, sub-splitting always happens due to thinner nanoplate thickness. It's worth noting that all the splitting events happen at the corners of the nanoplates. Combining real-time observation and Finite Element Method (FEM) simulation, we were able to quantify the Coulomb repulsive forces on the nanoplates, as shown in Fig. 2. Here, the electric field in the middle of the nanoplate is the smallest, and largest at the edges and corners. The magnitude of the Coulomb repulsive forces is also largest at the edges and corners, as shown by the arrows. This simulation offers a potential reason for the preferential splitting of the nanoplate at the corners. We further observed liquid-like features on the crystalline AgCl nanoplates. 
As shown in Fig. 3, a single crystalline $\mathrm{AgCl}$ nanoplate shrinks due to surface tension. The nanoplate maintains crystallinity while simultaneously behaves like a viscous liquid. We further estimated its selfdiffusivity by studying this surface tension induced shrinking, which is much larger than normal solids.

This work indicates that both the physical and chemical properties of nanosize materials are influenced by the interfacial confinement. Such a platform is ideal in exploring various interfacial phenomenon.

\section{References:}

[1] H. Zheng et al, Science 324 (2009), 1309.

[2] J. Lu et al, Nano Lett. 14 (2014), 2111.

[3] U. Mirsaidov et al, PNAS 109 (2012), 7187.

[4] K. Temsamani and K. Cheng, Sensor Actuat B 76 (2001), 551.

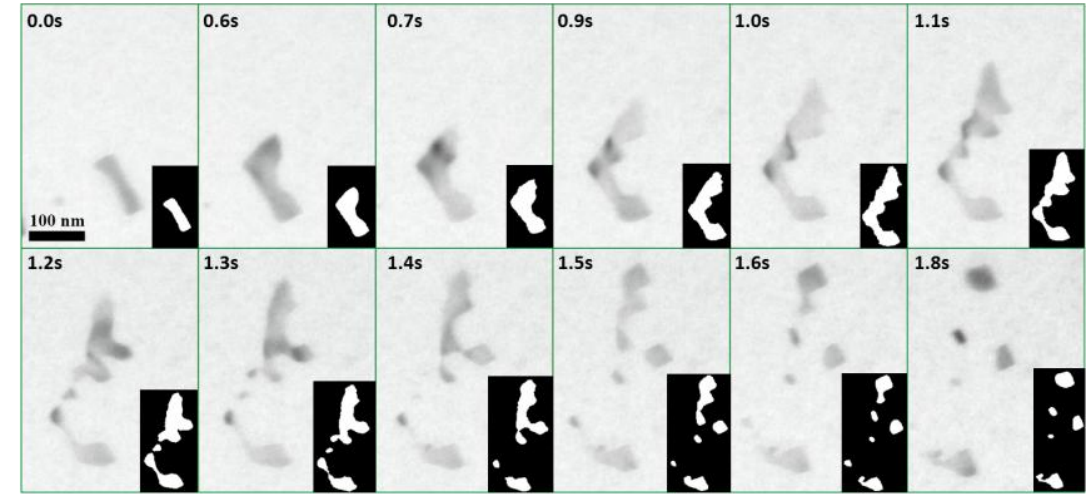

Figure 1. A typical nanoplate splitting process. Insets are their segmented 2 bit figures, respectively. The electron dose during this process was kept $13 \mathrm{e} /(\AA 2 \bullet \mathrm{s})$

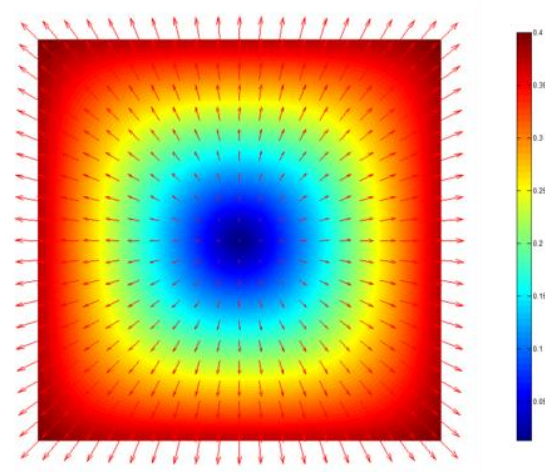

Figure 2. Simulated electric field (color) and Coulomb repulsive force (arrows).

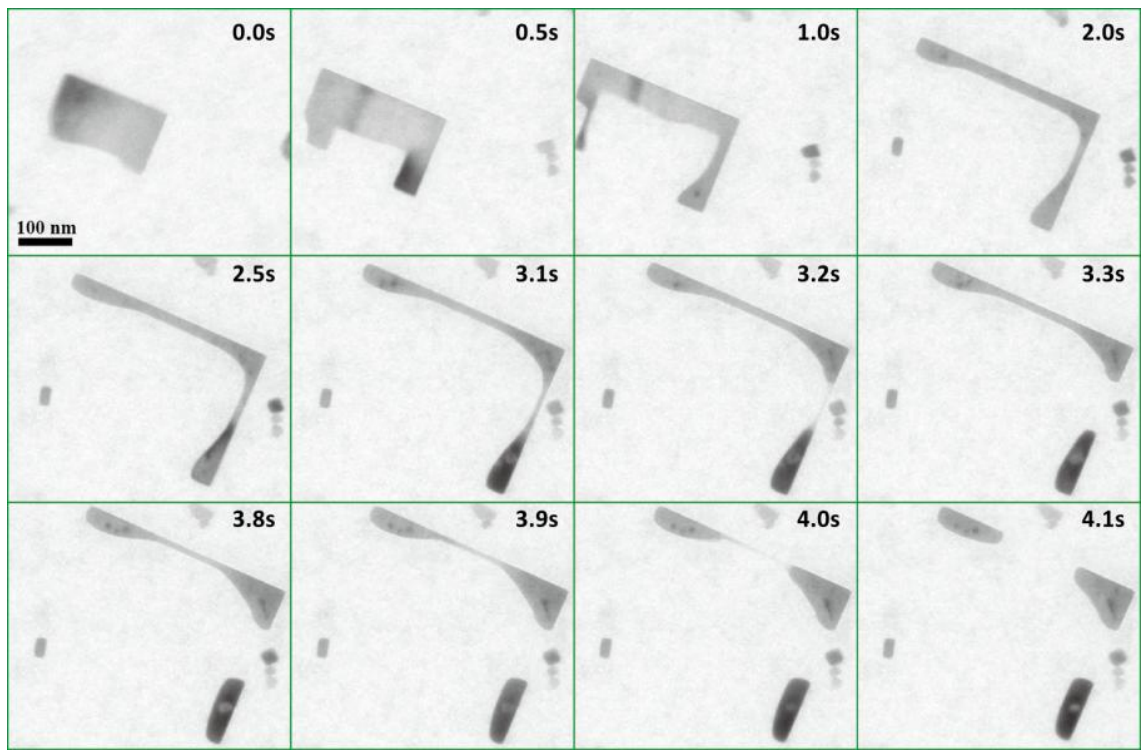

Figure 3. A single crystalline $\mathrm{AgCl}$ nanoplate shrinks due to surface tension. 